DOI: 10.1136/annrheumdis-2017-eular.6015

\section{AB0161 ANTIPHOSPHOLIPID ANTIBODIES DIFFERENTIALLY REGULATE THE EXPRESSION \& ACTIVITY OF THE LYSOSOMAL PROTEASES WITH EFFECTS UPON MONOCYTE AUTOPHAGY}

V.M. Ripoll-Nunez, A. Khawaja, I.P. Giles, A. Rahman. Division of Medicine, University College London, London, United Kingdom

Background: Antiphosholipid antibodies (aPLs) are known to activate monocytes in the pathogenesis of antiphospholipid syndrome (APS), although the precise mechanisms by which this activation occurs are not fully understood. We have recently identified several novel protein targets using a comprehensive proteomic analysis of human monocytes treated with lgG from patients with APS. Amongst these novel targets lysosomal proteases, including cathepsin B and cathepsin $\mathrm{D}$ were identified. These proteases are important in protein degradation, clearance of autolysosomes, apoptosis and autophagy. Dysregulation of these homeostatic cellular functions may be important in the exposure of autoantigens and pathogenesis of the APS. Therefore, we have now studied the effects of APS IgG upon the expression/activity of different cathepsins and their effects upon autophagy.

Objectives: Determine the effect of pathogenic aPL antibodies on monocyte autophagy and its association with the regulation of lysosomal activity.

Methods: Healthy monocytes were treated with $200 \mu \mathrm{g} / \mathrm{ml}$ of $\mathrm{lgG}$ purified from $(n=9)$ patients with APS or $(n=9)$ healthy control $(H C)$ IgG for 6 h. The expression of cathepsin B and cathepsin D were measured by western blotting. Activity assays for lysosomal proteases cathepsin D, cathepsin $B$ and cathepsin $L$ were performed using fluorescence based assays (RayBio ${ }^{\circledR}$ ). Intracellular proteolytic activity of monocytes was determined using DQ-BSA (Molecular probes) and flow cytometry analysis. Autophagy was induced by treating monocytes with $50 \mu \mathrm{g} / \mathrm{ml}$ GM-CSF for $14 \mathrm{~h}$.

Results: Consistent with our previous label free quantification mass spectrometry proteomic analysis, western blot analysis confirmed that levels of cathepsin B and cathepsin D were decreased in monocytes treated with APS IgG compared to $\mathrm{HC}$ IgG. Similarly, enzymatic assays revealed that cathepsin B and cathepsin $D$ activities were significantly reduced in monocytes treated with IgG from patients with APS compared to $\mathrm{HC}(\mathrm{p}=0.0188,0.0323)$. In contrast, levels of enzymatic activity of cathepsin $L$ were increased in monocytes treated with APS $\lg$ compared to $\mathrm{HC} \lg G(p=0.0106)$. To determine the effect of APS IgG on autophagy, we exposed healthy monocytes to $\lg G$ and induced autophagy by treating them with GM-CSF for $14 \mathrm{~h}$. Subsequently we tested the intracellular proteolytic activity with DQ-BSA. Stimulation of monocytes with APS IgG reduced the lysosomal activity of GM-CSF-treated monocytes whereas HC IgG had no effect, indicating that APS IgG disrupts lysosomal degradation during monocyte autophagy.

Conclusions: We found that IgG from patients with APS regulate the expression and activity of lysosomal proteases cathepsins $B / D$ and cathepsin $L$ in opposite directions. Activity of cathepsin $B$ and $D$ was down-regulated by exposure to $\mathrm{IgG}$ from patients with APS whereas cathepsin $\mathrm{L}$ was up-regulated. Furthermore, we found APS IgG disrupts lysosomal degradation during monocyte autophagy. Additional experiments are now underway to increase our understanding of how modulation of cathepsin activity and autophagy may be important in the pathogenesis of APS and provide new therapeutic targets.

Disclosure of Interest: None declared

DOI: 10.1136/annrheumdis-2017-eular.3417

\section{AB0162 SERUM LEVEL OF PROINFLAMMATORY CYTOKINES IS NEGATIVELY ASSOCIATED WITH FATIGUE IN PRIMARY SJÖGREN'S SYNDROME}

D. Gardiner ${ }^{1}$, W.D. Reid ${ }^{2}$, J.R. Tarn ${ }^{1}$, D. Lendrem ${ }^{1}$, N. Howard-Tripp ${ }^{1}$,

S. Bowman ${ }^{3}$, B. Griffiths ${ }^{4}$, S. Rushton ${ }^{2}$, W. .F. Ng ${ }^{1}$ on behalf of UKPSSR

${ }^{1}$ Institute of Cellular Medicine \& NIHR Newcastle Biomedical Research Centre;

${ }^{2}$ School of Biology, Newcastle University, Newcastle upon Tyne; ${ }^{3}$ Rheumatology, University Hospital Birmingham, Birmingham; ${ }^{4}$ Rheumatology, Freeman Hospital, Newcastle upon Tyne, United Kingdom

Background: We have previously described a model of fatigue in patients with primary Sjogren's syndrome (PSS) based on the levels of serum cytokines, pain and depression scores. Importantly, removal of cytokines from this model substantially reduced the accuracy suggesting that cytokines may have a key role in the biological basis of fatigue [1]. However, interpreting the model is complicated by the complexity of the immune system and the likely multiple interactions between numerous cytokines and other variables [2]. Structural equation modelling (SEM) is a statistical technique that allows for analysis of one or multiple independent variables with one or multiple dependent variables. SEM consists of two components - the structural model, which represents the relationships between the theoretical variables, and the measurement model, which are the relationships between the latent variables and their measures [3].

Objectives: To use SEM to test our hypothesis that the balance between pro-inflammatory and anti-inflammatory cytokines play an important role in determining severity of fatigue in patients with PSS.
Methods: We used Canonical Correpondence Analysis (CCA) to investigate the variation in cytokine expression across our spectrum of fatigue patietns to explore interactions and dependencies between cytokines. We then built a conceptual model based on the literature representing the likely relationships between fatigue and various proinflammatory and anti-inflammatory cytokines and other soluble molecules in the serum. This conceptual model was then challenged using serum data and fatigue scores of 161 PSS patients from the UK primary Sjogren's syndrome registry. Model fit was assessed using the Confirmatory Factor Index, the Root Mean Square Error of Association and the Standardised Root Mean Square Residual. We also analysed changes in fatigue scores over a period between 1-4 years.

Results: CCA revealed the first axis of ordination (CCA1) broadly correlates with fatigue, consists of many pro-inflammatory cytokines including TNF $\alpha, L T \alpha$ and IFN $\gamma$, IL17, which were negatively correlated with fatigue while IL-6 and MCP1, which were positively associated with increased fatigue severity. The second axis (CCA2) reflects a trend in cytokines which appear to relate to patients' age. Fatigue scores were largely stable over time and therefore data were not included in the SEM analysis. The main pro-inflammatory SEM model showed fatigue was negatively associated with pro-inflammatory cytokine activity $(p=0.019) ; \mathrm{IL}-10$ drove IP-10 $(p=0.000)$; and IL-10 was driven by IL-6 $(p=0.006)$ (Fig. 1)

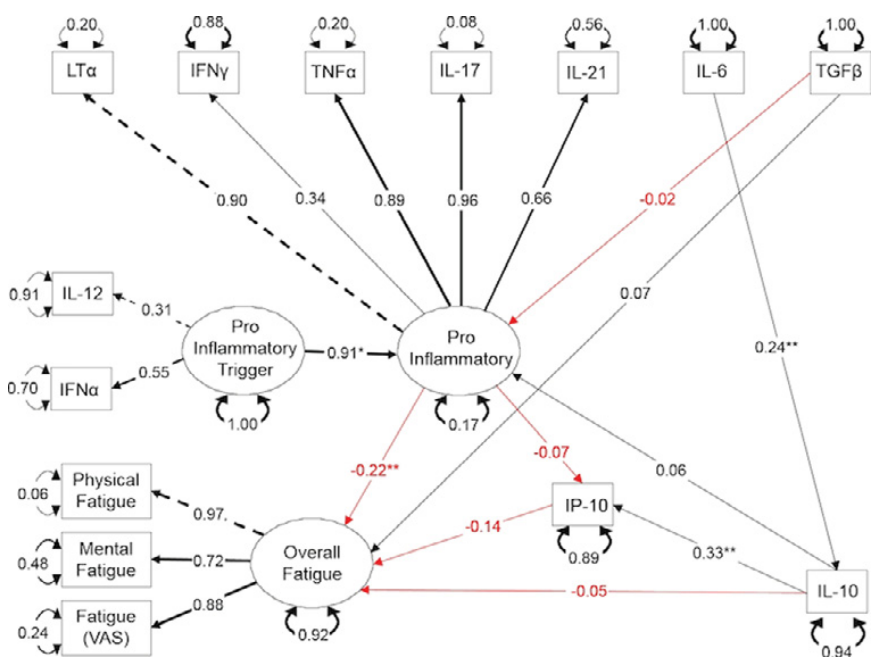

Conclusions: Chronic fatigue in PSS is negatively associated with many pro-inflammatory cytokines. We hypothesize that it reflects adaptive biological processes, which occurs after chronic exposure to inflammation in conditions such as PSS.

References:

[1] Howard-Tripp N, et al. RMD Open. 2016;2:e000282.

[2] Genser B, et al. BMC immunology. 2007;8:1.

[3] Beran TN \& Violato C. BMC Research Notes. 2010;3:267.

Acknowledgements: All UKPSSR participants.

Disclosure of Interest: D. Gardiner: None declared, W. Reid: None declared, J. Tarn: None declared, D. Lendrem: None declared, N. Howard-Tripp: None declared, S. Bowman Consultant for: Cellgene, Glenmark, GSK, Eli Lilly, Novartis, Roche, Takeda, UCB, B. Griffiths: None declared, S. Rushton: None declared, W.-F. Ng Consultant for: Pfizer, UCB, Medlmmune, Takeda and Sanofi

DOI: 10.1136/annrheumdis-2017-eular.5811

\section{AB0163 MESENCHYMAL STEM CELLS INDUCE CD1C+ TOLEROGENIC DENDRITIC CELLS IN HUMAN SYSTEMIC LUPUS ERYTHEMATOSUS VIA UP-REGULATING FLT-3 LIGAND}

X. Yuan, D. Wang, Z. Zhang, Q. Wang, W. Li, X. Tang, W. Chen, G. Yao, L. Sun. Department of Rheumatology and Immunology, The Affiliated Drum Tower hospital of Nanjing University Medical School, Nanjing, China

Background: $\mathrm{CD}_{1 \mathrm{c}^{+}}$tolerogenic dendritic cells (DCs) play important roles in the induction of peripheral tolerance and control of adaptive immune response. Umbilical cord (UC)-derived mesenchymal stem cells (MSCs) exhibit immunoregulation effects in systemic lupus erythematosus (SLE). However, the underlying immunosuppression mechanism of MSCs via tolerogenic DCs in SLE remains largely unknown.

Objectives: The aim of this study was to examine tolerogenic DCs levels in SLE patients, and to further investigate the mechanism of MSCs in the regulation of tolerogenic DCs.

Methods: Tolerogenic DCs were isolated as Lin (CD3/19/56/14)- HLA $\mathrm{DR}^{+} \mathrm{CD} 11 \mathrm{c}^{+} \mathrm{CD} 1 \mathrm{c}^{+}$from peripheral blood mononuclear cells (PBMCs). Levels of tolerogenic DCs were determined by flow cytometry, and serum concentration of FIt-3 ligand (FLT3L) were determined by ELISA from 17 healthy controls and 25 SLE patients. Eight SLE patients were given UC MSCs infusions. We compared the levels of tolerogenic DCs and serum FLT3L before and 24 hours after UC 